Volume 360, Number 3, March 2008, Pages 1581-1599

S 0002-9947(07)04280-8

Article electronically published on October 22, 2007

\title{
HEEGNER DIVISORS IN THE MODULI SPACE OF GENUS THREE CURVES
}

\author{
MICHELA ARTEBANI
}

\begin{abstract}
S. Kondo used periods of $K 3$ surfaces to prove that the moduli space of genus three curves is birational to an arithmetic quotient of a complex 6-ball. In this paper we study Heegner divisors in the ball quotient, given by arithmetically defined hyperplane sections of the ball. We show that the corresponding loci of genus three curves are given by hyperelliptic curves, singular plane quartics and plane quartics admitting certain rational "splitting curves".
\end{abstract}

\section{INTRODUCTION}

The degree four cyclic cover of the projective plane branched along a smooth plane quartic is a $K 3$ surface endowed with an automorphism group $G \cong \mathbb{Z}_{4}$. This simple geometric construction relates the moduli space $\mathcal{M}_{3}$ of genus three curves to a moduli space $\mathcal{M}$ of polarized $K 3$ surfaces. A generator $\sigma$ for $G$ can be chosen such that the period point of the polarized $K 3$ surface $(X, \sigma)$ belongs to the $i$-eigenspace $W_{\mathbb{C}}$ of $\sigma^{*}$ in $H^{2}(X, \mathbb{C})$. This implies that the period domain is a six dimensional complex ball $B \subset \mathbb{P}\left(W_{\mathbb{C}}\right)$ and the moduli space $\mathcal{M}$ is obtained by taking the quotient of $B$ by the action of an arithmetic group $\Gamma$. The correspondence between genus three curves and polarized $K 3$ surfaces is thus described by a period map

$$
\mathcal{P}: \mathcal{M}_{3} \backslash \mathcal{M}_{3}^{h} \longrightarrow \mathcal{M} \cong B / \Gamma,
$$

where $\mathcal{M}_{3}^{h}$ denotes the hyperelliptic locus. In fact, in [7] S. Kondo proves that this map is birational and induces an isomorphism:

$$
\mathcal{M}_{3} \backslash \mathcal{M}_{3}^{h} \cong \mathcal{M} \backslash\left(\mathcal{D}_{n} \cup \mathcal{D}_{h}\right),
$$

where $\mathcal{D}_{n}, \mathcal{D}_{h}$ are two irreducible divisors, called mirrors.

The $\mathbb{Q}$-vector space $H^{2}(X, \mathbb{Q})$ and the action of $\sigma$ naturally give a vector space $W$ over $k=\mathbb{Q}[i]$ such that $W_{\mathbb{C}}=W \otimes_{k} \mathbb{C}$. An interesting class of divisors in $\mathcal{M}$ is then given by quotients of hyperplane sections of $B$ defined over $k$. These divisors are again arithmetic quotients of a (five dimensional) complex ball and are called Heegner divisors. This kind of divisor has been introduced and studied in a more general setting in [8].

The first examples of Heegner divisors are the two mirrors $\mathcal{D}_{n}, \mathcal{D}_{h}$. In [7] it is proved that the generic points in the mirrors correspond, via the period map $\mathcal{P}$, to a plane quartic with a node and to a smooth hyperelliptic genus three curve

Received by the editors October 12, 2005 and, in revised form, February 20, 2006.

2000 Mathematics Subject Classification. Primary 14J10, 14J28, $14 \mathrm{H} 10$.

Key words and phrases. Genus three curves, splitting curves, K3 surfaces, Heegner divisors.

This work was partially supported by PRIN 2003: Spazi di moduli e teoria di Lie; GNSAGA. 
respectively. The aim of this paper is to describe all Heegner divisors in terms of genus three curves. We prove indeed that any Heegner divisor which is not a mirror can be interpreted as the locus of plane quartics admitting a splitting curve i.e. an irreducible plane curve whose inverse image by the degree four cyclic cover is the union of four distinct curves on the $K 3$ surface. More precisely, we show that the splitting curve can be chosen to be rational. The simplest example of Heegner divisor of this kind is the divisor of plane quartics with a hyperflex line.

The paper is organized as follows.

In the first section we introduce some basic notations for genus three curves, Del Pezzo surfaces and $K 3$ surfaces.

The geometric construction by Kondō and its main theorem are recalled in the second section.

In the third section we define Heegner divisors and show how they are connected to the problem of embedding a rank two lattice in a non-unimodular lattice. In fact, we introduce two natural invariants associated to such embeddings: the type $n \in \mathbb{Z}, n>0$ and the index $m \in\{1,2\}$. We also provide an existence result for Heegner divisors with fixed type and index.

In the following section Heegner divisors are interpreted in terms of genus three curves. The main theorem states that the generic point in a Heegner divisor of type $n>1$ corresponds to a smooth plane quartic having a splitting rational curve with smooth preimages in the Del Pezzo surface. Moreover, we give the minimal degree of such a splitting curve as a function of $n$ and $m$ (it turns out that it is even if $m=1$ and odd if $m=2$ ).

Some examples of Heegner divisors are provided in the last section for splitting curves of degree 1,2 and 3.

\section{Notation AND PRELIMINARIES}

In this section we introduce the three geometric objects of main interest in this paper and fix the relative notations: genus three curves, Del Pezzo surfaces and $K 3$ surfaces. Our main references are [9], [4] and [2].

Let $\mathcal{M}_{3}$ be the coarse moduli variety parametrizing isomorphism classes of smooth genus three curves. We denote by $\mathcal{M}_{3}^{h}$ the hyperelliptic divisor in $\mathcal{M}_{3}$.

A Del Pezzo surface is a smooth surface with an ample anti-canonical bundle. These surfaces can be equivalently defined as the blowing up of $\mathbb{P}^{2}$ in a set of $m \leq 8$ distinct points "in general position". We are interested in Del Pezzo surfaces of degree two i.e. the blowing up of $\mathbb{P}^{2}$ in seven points $p_{1}, \ldots, p_{7}$. In this case, a natural basis for the Picard lattice of the surface $S$ is given by

$$
\operatorname{Pic}(S)=\left\langle e_{0}, e_{1}, \ldots, e_{7}\right\rangle,
$$

where $e_{0}$ is the pull-back of the hyperplane bundle of $\mathbb{P}^{2}$ and $e_{i}$ is the exceptional divisor corresponding to $p_{i}, i=1, \ldots, 7$. In this basis, the anti-canonical bundle of $S$ is given by:

$$
-k=3 e_{0}-\left(e_{1}+\cdots+e_{7}\right) .
$$

The morphism $\phi_{(-k)}: S \longrightarrow \mathbb{P}^{2}$ associated to the anti-canonical linear system is a double cover of the plane branched along a smooth plane quartic curve $Q(S)$. There are exactly 56 effective divisors in $\operatorname{Pic}(S)$ with self-intersection -1 , given by the preimages of the 28 bitangents of $Q(S)$. Now consider the lattice:

$$
R=k^{\perp}=\{x \in \operatorname{Pic}(S):(x, k)=0\},
$$


where $($,$) denotes the intersection form on \operatorname{Pic}(S)$. This a root lattice of type $E_{7}$ and a root basis is given by:

$$
\begin{gathered}
\alpha_{0}=e_{0}-\left(e_{1}+e_{2}+e_{3}\right), \\
\alpha_{i}=e_{i}-e_{i+1}, \quad i=1, \ldots, 6 .
\end{gathered}
$$

We denote by $W\left(E_{7}\right)$ the associated Weyl group.

Let $i$ be the covering involution of $\phi_{(-k)}$; then the induced involution $i^{*}$ on $\operatorname{Pic}(S)$ generates the center of the Weyl group $W\left(E_{7}\right)$ (see 4 ., Ch.VII, [4]). It is easy to see that

$$
\begin{gathered}
i^{*}\left(e_{i}\right)=-k-e_{i}, \quad i=1, \ldots, 7, \\
i^{*}\left(e_{0}\right)=-3 k-e_{0} .
\end{gathered}
$$

The eigenlattices $H_{ \pm}$of $i^{*}$ relative to \pm 1 are given by

$$
H_{+}=\langle k\rangle, H_{-}=R \text {. }
$$

Let $X$ be a $K 3$ surface i.e. a surface with $q(X)=0$ and trivial canonical bundle. We denote by $\omega_{X}$ a generator for the vector space $H^{2,0}(X)$ of holomorphic twoforms on $X$, with $\operatorname{Pic}(X)$ the Picard lattice and with $T(X)$ the transcendental lattice of $X$. We recall that the cohomology group $H^{2}(X, \mathbb{Z})$ is an even unimodular lattice isometric to $L_{K 3}=U^{\oplus 3} \oplus E_{8}^{\oplus 2}$. The intersection form on $L_{K 3}$ is denoted by $($,$) .$

\section{The MOdel By Kondō}

2.1. Geometric construction. We briefly recall the geometric construction introduced by Kondō in [7]. Let $C$ be a smooth quartic curve in $\mathbb{P}^{2}$ defined by a homogeneous polynomial $f \in \mathbb{C}[x, y, z]$ of degree 4 :

$$
C=\left\{(x, y, z) \in \mathbb{P}^{2}: f(x, y, z)=0\right\} .
$$

Consider the 4:1 cyclic cover of $\mathbb{P}^{2}$ branched along the divisor $C$ :

$$
\pi: X_{C} \stackrel{4: 1}{\longrightarrow} \mathbb{P}^{2} \text {. }
$$

The surface $X_{C}$ is a $K 3$ surface with a degree four polarization; in coordinates it can be given by:

$$
X_{C}=\left\{(x, y, z, t) \in \mathbb{P}^{3}: t^{4}=f(x, y, z)\right\} .
$$

Let $\sigma$ be a generator for the covering transformation group of $\pi$ and $\tau=\sigma^{2}$. We can assume that:

$$
\sigma(x, y, z, t)=(x, y, z, i t) .
$$

Then the morphism $\pi$ factors naturally through the double cover of $\mathbb{P}^{2}$ branched along $C$ :

$$
\pi_{1}: S_{C} \stackrel{2: 1}{\longrightarrow} \mathbb{P}^{2},
$$

where $S_{C}=X_{C} /\langle\tau\rangle$ is a Del Pezzo surface of degree 2 and $\pi_{1}=\phi_{(-k)}$ is the morphism associated to the anti-canonical linear system of $S_{C}$. The geometry of the above construction is then described by the following commutative diagram:

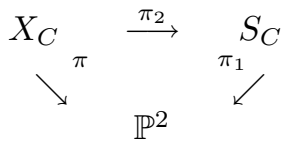

The $K 3$ surface $X_{C}$ is $L_{+}$-polarized, where $L_{+}$denotes the pull-back of $\operatorname{Pic}\left(S_{C}\right)$ and the isometry $\sigma^{*}$ induced by $\sigma$ on the cohomology lattice acts as a 4 -th root 
of unity on the vector space of holomorphic two-forms. In other words, the period point of $X_{C}$ belongs to $\mathbb{P}\left(W_{\mathbb{C}}\right)$, where $W_{\mathbb{C}}$ is an eigenspace of $\sigma^{*}$ in $L_{+}^{\perp} \otimes \mathbb{C}$. In fact, if $L_{-}=L_{+}^{\perp}$, then $W_{\mathbb{C}} \cong L_{-} \otimes_{\mathbb{Z}} \mathbb{R}$ and the action of $\sigma^{*}$ gives to $W=L_{-} \otimes_{\mathbb{Z}} \mathbb{Q}$ the structure of a vector space over $k=\mathbb{Q}[i]$.

The vector space $W_{\mathbb{C}}$ is equipped with the hermitian form

$$
\varphi(z, w)=(z, \bar{w})
$$

of signature $(1,6)$. The period domain $\mathcal{M}$ for polarized $K 3$ surfaces $\left(X_{C}, \sigma^{*}\right)$ is then an arithmetic quotient of the six dimensional complex ball $B$ in $\mathbb{P}\left(W_{\mathbb{C}}\right)$ defined by $\varphi(z, z)<0$ :

$$
\begin{gathered}
\mathcal{M}=B / \Gamma \\
\Gamma=\left\{\gamma \in O\left(L_{-}\right): \gamma \circ \sigma^{*}=\sigma^{*} \circ \gamma\right\} \cong U(\varphi) \cap M(7, \mathbb{Z}[i]) .
\end{gathered}
$$

The above construction defines a period map:

$$
\mathcal{P}: \mathcal{M}_{3} \backslash \mathcal{M}_{3}^{h} \longrightarrow \mathcal{M},
$$

where $\mathcal{M}_{3}^{h}$ denotes the hyperelliptic locus. In fact, the image of the period map lies in the complement of two divisors $\mathcal{D}_{n}, \mathcal{D}_{h}$, called mirrors, corresponding to non-ample polarized $K 3$ surfaces. The main result in [7] is:

Theorem 2.1 (S. Kondō, Theorem 2.5, [7]). The period map gives an isomorphism:

$$
\mathcal{P}: \mathcal{M}_{3} \backslash \mathcal{M}_{3}^{h} \longrightarrow \mathcal{M} \backslash\left(\mathcal{D}_{n} \cup \mathcal{D}_{h}\right) .
$$

2.2. Isometries. Given a lattice $L$ we will denote with $r(L)$ its rank and with $\left(s_{+}, s_{-}\right)$its signature. Moreover let $A_{L}=L^{*} / L$ be the discriminant group, $\ell(L)$ be the minimal number of generators of $A_{L}$ and $q_{L}$ be the quadratic form on it. We also introduce the invariant

$$
\delta_{L}= \begin{cases}0 & \text { if } q_{L}(x) \in \mathbb{Z} \text { for all } x \in A_{L} \\ 1 & \text { otherwise }\end{cases}
$$

We now describe the action of covering transformations on the cohomology lattice. Let $\tau^{*}$ be the involution on $H^{2}\left(S_{C}, \mathbb{Z}\right)$ induced by $\tau$; its eigenlattices are given by:

$$
L_{ \pm}=\left\{x \in H^{2}\left(X_{C}, \mathbb{Z}\right): \tau^{*}(x)= \pm x\right\} .
$$

In fact, the invariant lattice $L_{+}$is the Picard lattice of the generic $K 3$ surface $X_{C}$ and $L_{-}=L_{+}^{\perp}$. The isomorphism classes of the eigenlattices can be easily computed:

\section{Lemma 2.2 .}

$$
L_{+} \simeq\langle 2\rangle \oplus A_{1}^{\oplus 7}, \quad L_{-} \simeq\langle 2\rangle^{\oplus 2} \oplus D_{4}^{\oplus 3} .
$$

Proof. From Theorem 4.2.2, [10] and Theorem 3.6.2, [11] it follows that $L_{+}$is a 2-elementary lattice of signature $(1,7)$ with $r\left(L_{+}\right)=\ell\left(L_{+}\right)=8$ and $\delta\left(L_{+}\right)=1$. Hence obviously: $r\left(L_{-}\right)=14, \ell\left(L_{-}\right)=8$ and $\delta\left(L_{-}\right)=1$. By Theorem 3.6.2, [11] the isomorphism classes of the two lattices are determined uniquely by the set of invariants $\left(s_{+}, s_{-}, \ell, \delta\right)$. Hence it is enough to check that the lattices in the right hand sides have the same set of invariants; this follows easily since $\ell\left(A_{1}\right)=\delta\left(A_{1}\right)=$ 1 and $\ell\left(D_{4}\right)=2, \delta\left(D_{4}\right)=0$. For the proof of the first isomorphism see also $\S 2$, [7]. 
From Lemma 2.2 it follows that $L_{+}=\pi_{2}^{*} \operatorname{Pic}\left(S_{C}\right)$. For $x \in \operatorname{Pic}\left(S_{C}\right)$ we define:

$$
\tilde{x}=\pi_{2}^{*}(x) \in L_{+} .
$$

In particular, fixing a blowing-up morphism $b: S_{C} \longrightarrow \mathbb{P}^{2}$ with the notation in section 1 , a basis for $L_{+}$is given by:

$$
\tilde{e}_{0}, \tilde{e}_{1}, \ldots, \tilde{e}_{7} .
$$

The action of $\sigma^{*}$ on $L_{+}$is clearly the pull-back along $\pi_{2}$ of the action of the involution $i^{*}$ on $\operatorname{Pic}(S)$ defined in the previous section. In particular, let $\tilde{R}=\pi_{2}^{*}(R)$ and $\widetilde{H}_{ \pm}$be the eigenlattices of $\sigma^{*}$ on $L_{+}$.

From the remarks in section 1 there immediately follows:

\section{Lemma 2.3.}

$$
\widetilde{H}_{+}=\langle\tilde{k}\rangle \cong\langle 4\rangle, \widetilde{H}_{-}=\tilde{R} \cong E_{7}(2) .
$$

Moreover, the action of the Weyl group $W\left(E_{7}\right)$ lifts to $L_{+}$giving a subgroup:

$$
W\left(E_{7}\right) \subset O\left(L_{+}\right)
$$

of isometries commuting with $\sigma^{*}$.

A natural basis for $L_{-}$is given by:

$$
t_{1}, t_{2}, f_{1}^{1}, \ldots, f_{4}^{1}, f_{1}^{2}, \ldots, f_{4}^{2}, f_{1}^{3}, \ldots, f_{4}^{3},
$$

where $t_{1}, t_{2}$ is a root basis for $\langle 2\rangle^{\oplus 2}$ and $f_{1}^{i}, \ldots, f_{4}^{i}$ is a root basis for the $i$-th copy of $D_{4}$. Then we have

Lemma 2.4. There exists an isomorphism $L_{-} \cong\langle 2\rangle^{\oplus 2} \oplus D_{4}^{\oplus 3}$ such that the action of the isometry $\sigma^{*}$ on $L_{-}$preserves $\langle 2\rangle^{\oplus 2}$ and each copy of $D_{4}$. In fact it is given by the matrix $J_{1} \oplus J_{2}^{\oplus 3}$, where:

$$
J_{1}=\left(\begin{array}{cc}
0 & 1 \\
-1 & 0
\end{array}\right), J_{2}=\left(\begin{array}{cccc}
0 & 1 & 0 & 0 \\
-1 & 0 & 0 & 0 \\
1 & 0 & 1 & 1 \\
-1 & 1 & 2 & 1
\end{array}\right)
$$

with respect to the previously defined basis of $L_{-}$.

Proof. See [1].

The discriminant groups of the eigenlattices of $\tau^{*}$ are given by (Proposition 3.2.2, $[10]):$

$$
A_{L_{+}} \cong A_{L_{-}} \cong \mathbb{Z}_{2}^{\oplus 8}
$$

In fact, we consider the natural bases:

$$
\begin{aligned}
& A_{L_{+}}=\left\langle\tilde{e}_{0} / 2, \tilde{e}_{1} / 2, \ldots, \tilde{e}_{7} / 2\right\rangle, \\
& A_{L_{-}}=\left\langle t_{1} / 2, t_{2} / 2, \alpha_{1}^{i}, \alpha_{2}^{i}, i=1,2,3\right\rangle,
\end{aligned}
$$

where $\alpha_{1}^{i}, \alpha_{2}^{i}$ generate the discriminant group of the $i$-th copy of $D_{4}$ in $L_{-}$. By Proposition 1.6.1, [10], the primitive embedding of $L_{+}$in $L_{K 3}$ is determined by an isomorphism

$$
\gamma: A_{L_{+}} \longrightarrow A_{L_{-}} \text {with } q_{L_{-}} \circ \gamma=q_{L_{+}}
$$


Lemma 2.5. The isomorphism $\gamma$ is given by the matrix

$$
M(\gamma)=\left(\begin{array}{llllllll}
0 & 1 & 1 & 1 & 1 & 1 & 1 & 1 \\
1 & 0 & 0 & 0 & 0 & 0 & 0 & 0 \\
1 & 0 & 1 & 0 & 0 & 0 & 0 & 0 \\
1 & 0 & 0 & 1 & 0 & 0 & 0 & 0 \\
1 & 0 & 0 & 0 & 1 & 0 & 0 & 0 \\
1 & 0 & 0 & 0 & 0 & 1 & 0 & 0 \\
1 & 0 & 0 & 0 & 0 & 0 & 1 & 0 \\
1 & 0 & 0 & 0 & 0 & 0 & 0 & 1
\end{array}\right)
$$

up to the action of $O\left(A_{L_{+}}\right)$(with respect to the previously defined bases).

Proof. The primitive embedding of $L_{+}$in $L_{K 3}$ is unique by Theorem 1.14.4, [10]. Hence, by Proposition 1.6.1, [10], the isomorphism $\gamma$ is unique up to the action of the image of the natural homomorphism

$$
\psi: O\left(L_{+}\right) \longrightarrow O\left(A_{L_{+}}\right) .
$$

In fact, by Theorem 1.14.2, [10], the homomorphism $\psi$ is surjective. Hence, it is enough to check that $M(\gamma)$ defines an isomorphism preserving the quadratic forms.

The following result describes all vectors in $A_{L_{+}}$with assigned self-intersection with respect to the quadratic form $q_{L_{+}}$and the action of $\sigma^{*}$ on them:

Lemma 2.6 (Lemma 2.1, [7]). The vectors in $A_{L_{+}}$can be divided in four classes accordingly to their self-intersection:

$$
\begin{aligned}
& C_{1}=\left\{x \in A_{L_{+}} \mid q_{L_{+}}(x)=-1 / 2\right\}, \\
& C_{2}=\left\{x \in A_{L_{+}} \mid q_{L_{+}}(x)=1 / 2\right\}, \\
& C_{3}=\left\{x \in A_{L_{+}} \mid q_{L_{+}}(x)=1\right\}, \\
& C_{4}=\left\{x \in A_{L_{+}} \mid q_{L_{+}}(x)=0\right\} .
\end{aligned}
$$

- An element in $C_{1}$ is represented uniquely by one of the following 56 vectors:

$$
\begin{gathered}
\frac{\tilde{e}_{i}}{2}, \frac{\tilde{k}-\tilde{e}_{i}}{2}, \quad 1 \leq i \leq 7, \\
\frac{\tilde{e_{0}}-\tilde{e_{i}}-\tilde{e_{j}}}{2}, \frac{\tilde{k}-\tilde{e_{0}}+\tilde{e}_{i}+\tilde{e}_{j}}{2}, \quad 1 \leq i<j \leq 7 .
\end{gathered}
$$

- An element in $C_{2}$ is represented uniquely by one of the following 72 vectors:

$$
\begin{gathered}
\frac{\tilde{e}_{0}}{2}, \frac{\tilde{k}-\tilde{e_{0}}}{2}, \\
\frac{\tilde{e}_{i}+\tilde{e}_{j}+\tilde{e}_{k}}{2}, \frac{\tilde{k}-\tilde{e}_{i}-\tilde{e}_{j}-\tilde{e}_{k}}{2}, \quad 1 \leq i<j<k \leq 7 .
\end{gathered}
$$

- An element in $C_{3}$ is represented uniquely by one of the following 64 vectors:

$$
\begin{gathered}
\frac{\tilde{k}}{2}, \frac{\tilde{e}_{i}-\tilde{e}_{j}}{2}, \quad 1 \leq i<j \leq 7, \\
\frac{\tilde{e}_{0}-\tilde{e}_{i}-\tilde{e}_{j}-\tilde{e}_{k}}{2}, \quad 1 \leq i<j<k \leq 7, \\
\frac{2 \tilde{e}_{0}-\sum_{i} \tilde{e}_{i}+\tilde{e}_{j}}{2}, \quad 1 \leq j \leq 7 .
\end{gathered}
$$


- An element in $C_{4}$ is represented uniquely by one of the following 64 vectors:

$$
\begin{gathered}
0, \frac{\tilde{k}+\tilde{e}_{i}-\tilde{e}_{j}}{2}, \quad 1 \leq i<j \leq 7, \\
\frac{\tilde{k}+\tilde{e}_{0}-\tilde{e}_{i}-\tilde{e}_{j}-\tilde{e}_{k}}{2}, \quad 1 \leq i<j<k \leq 7, \\
\frac{\tilde{k}+2 \tilde{e}_{0}-\sum_{i} \tilde{e}_{i}+\tilde{e}_{j}}{2}, \quad 1 \leq j \leq 7 .
\end{gathered}
$$

The isometry $\sigma^{*}$ acts as:

$$
\sigma^{*}(x)=\left\{\begin{array}{cl}
\tilde{k} / 2-x & \text { if } x \in C_{1} \cup C_{2}, \\
x & \text { if } x \in C_{3} \cup C_{4} .
\end{array}\right.
$$

Let

$$
N_{+}=\left\{x \in L_{+}^{*}: q_{L_{+}}(x) \in \mathbb{Z} / 2 \mathbb{Z}\right\}
$$

Notice that:

$$
N_{+} / L_{+}=\left\langle\tilde{k} / 2, \tilde{\alpha}_{1} / 2, \ldots, \tilde{\alpha}_{6} / 2\right\rangle .
$$

We denote by $O\left(A_{L_{+}}\right)$the group of automorphisms of $A_{L_{+}}$respecting the discriminant quadratic form $q_{L_{+}}$. Then we have

Lemma 2.7. The natural homomorphism $W\left(E_{7}\right) \longrightarrow O\left(A_{L_{+}}\right)$is surjective. Moreover, the action of $O\left(A_{L_{+}}\right)$on vectors in Lemma 2.6 is transitive on classes $C_{1}$ and $C_{2}$ and has two orbits on classes $C_{3}$ and $C_{4}$.

Proof. The first assertion follows from Lemma 2.3, [7]. The elements of $C_{1}$ are of the form $\tilde{x} / 2$ where $x$ is the class of a $(-1)$-curve on the Del Pezzo surface $S$. The Weyl group $W\left(E_{7}\right)$ acts transitively on $(-1)$-curves (see for example Lemma 4 , Ch. $\mathrm{V},[4])$, hence the action of $O\left(A_{L_{+}}\right)$is transitive on $C_{1}$. Now consider the elements in the class $C_{2}$ :

$$
\tilde{e}_{0} / 2, v_{i j k}=\left(\tilde{e}_{i}+\tilde{e}_{j}+\tilde{e}_{k}\right) / 2 .
$$

Let $\alpha_{i j k}=\tilde{e}_{0}-v_{i j k}$, then:

$$
s_{\alpha_{i j k}}\left(v_{i j k}\right)=3 \tilde{e}_{0}-2 v_{i j k} / 2=\tilde{e}_{0} / 2\left(\bmod L_{+}\right) .
$$

Similarly, two vectors $v_{i j k}$ and $v_{i^{\prime} j^{\prime} k^{\prime}}$ are interchanged by the isometry obtained as a composition of the three reflections with respect to $e_{i}-e_{i^{\prime}}, e_{j}-e_{j^{\prime}}, e_{k}-e_{k^{\prime}}$. This gives the assertion for $C_{2}$.

The element $\tilde{k} / 2$ in the class $C_{3}$ is preserved by the Weyl group. The other 63 classes are of the form $\tilde{x} / 2$ where $x$ is a (positive) root of $E_{7}$. Since $W\left(E_{7}\right)$ acts transitively on roots (see for example [4]), the action is transitive on $C_{3}$.

The elements in $C_{4}$ are obtained from those in $C_{3}$ by adding $\tilde{k} / 2$, hence the assertion follows easily.

\section{HEEGNER DIVISORS AND EMBEDDINGS}

Definition 3.1. Let $r \in L_{-}$be a primitive vector and let $H_{r}$ be the hyperplane section of $B$ orthogonal to $r$ :

$$
H_{r}=\{z \in B:(z, r)=0\} .
$$

The Heegner divisor $\mathcal{D}_{r}$ associated to $r$ is the image of $H_{r}$ in $\mathcal{M}$. A Heegner divisor $\mathcal{D}_{r}$ is of type $n$ if $r^{2}=-2 n$. 
Remark 3.2. i) For $r \in L_{-}$let $z(r)=r-i \sigma^{*}(r)$. Then $z(r) \in W$ and

$$
H_{r}=\{z \in W: \varphi(z, z(r))=0\},
$$

where $\varphi$ is the hermitian form on $W$ defined in section 2. This implies that a Heegner divisor can be equivalently defined as the image in $\mathcal{M}$ of a hyperplane section of $B$ defined over $k=\mathbb{Q}[i]$. In the language of [8], Heegner divisors are quotients of an arithmetically defined arrangement of hyperplanes in the ball.

ii) It can be easily seen that $H_{r}=H_{\sigma^{*}(r)}$ and $H_{r}=\emptyset$ if $n \leq 0$.

We have a lattice isomorphism:

$$
\Lambda_{r}=\left\langle r, \sigma^{*}(r)\right\rangle \cong A_{1}(n)^{\oplus 2} .
$$

Hence, giving a Heegner divisor of type $n \geq 1$ in $\mathcal{M}$ is equivalent to assigning a primitive embedding of the lattice $A_{1}(n)^{\oplus 2}$ in $L_{-}$up to the action of the group $\Gamma$. Given such an embedding, let $\Lambda_{r}^{\perp}$ be the orthogonal complement of $\Lambda_{r}$ in $L_{-}$and $P$ be the orthogonal complement of $\Lambda_{r}^{\perp}$ in $L_{K 3}$. We have an embedding

$$
L_{+} \oplus \Lambda_{r} \subset P
$$

with finite quotient group

$$
M_{r}=P /\left(L_{+} \oplus \Lambda_{r}\right) .
$$

We now start describing the structure of the group $M_{r}$.

Proposition 3.3. Let $r \in L_{-}$and $M_{r}$ as above. Then:

a) if $n$ is even, then $M_{r} \cong \mathbb{Z}_{2}$ and $\left(r, L_{-}\right)=\mathbb{Z}$;

b) if $n$ is odd, then:

$$
M_{r} \cong\left\{\begin{array}{lll}
\mathbb{Z}_{2} \oplus \mathbb{Z}_{2} & \text { if } & \left(r, L_{-}\right)=2 \mathbb{Z} \\
\mathbb{Z}_{2} & \text { if } & \left(r, L_{-}\right)=\mathbb{Z} .
\end{array}\right.
$$

Proof. The group $M_{r}$ has a natural embedding in $A_{L_{+}} \oplus A_{\Lambda_{r}}$. Moreover, since the embedding of $\Lambda_{r}$ in $P$ is primitive, the projections on the two factors are isomorphisms (see [11]):

$$
M_{r} \cong M_{1} \subset A_{L_{+}}, M_{r} \cong M_{2} \subset A_{\Lambda_{r}}
$$

such that

$$
q_{L_{+\mid M_{1}}}=-q_{\Lambda_{r \mid M_{2}}}
$$

Since

$$
A_{L_{+}} \cong \mathbb{Z}_{2}^{\oplus 8}, A_{\Lambda_{r}} \cong \mathbb{Z}_{2 n}^{\oplus 2}
$$

it follows that:

$$
M_{r} \cong \mathbb{Z}_{2}^{\oplus m_{r}}, \quad 0 \leq m_{r} \leq 2 .
$$

We first prove that the case $m_{r}=0$ does not appear i.e. $L_{+} \oplus \Lambda_{r}$ cannot be primitively embedded in $L_{K 3}$. This means that for any embedding $L_{+} \oplus \Lambda_{r} \hookrightarrow L_{K 3}$ there is a primitive element $(x, y) \in L_{+} \oplus \Lambda_{r}$ such that $(x, y) / 2 \in L_{K 3}$. For this, it suffices to prove that, for some $y \in \Lambda_{r}, y / 2$ is not trivial in $A_{L_{-}}$, since in this case $($ see $\S 2.2)$ :

$$
\gamma^{-1}(y / 2)+y / 2 \in L_{K 3} .
$$

Note that a vector in $D_{4}$ can be written as

$$
d=a_{1} e_{1}+\ldots+a_{4} e_{4}, \quad \sum a_{i} \equiv 0(\bmod 2),
$$


where $\left(e_{i} \cdot e_{j}\right)=-\delta_{i j}$. If $d$ is primitive in $D_{4}$ (in particular not all $a_{i}$ 's are even), then:

a) if $a_{i}$ is odd for $i=1, \ldots, 4$, then $d / 2$ is non-trivial in $A_{D_{4}}$;

b) if $a_{1}, a_{3}$ are odd and $a_{2}, a_{4}$ are even, then $\left(d+\sigma^{*}(d)\right) / 2$ has integer coefficients but either 1 or 3 of the coefficients are odd, hence it is a non-trivial element in $A_{D_{4}}$;

c) if $a_{1}, a_{2}$ are odd and $a_{3}, a_{4}$ are even, then all coefficients of $\left(d+\sigma^{*}(d)\right)$ are odd integers, so $\left(d+\sigma^{*}(d)\right) / 2$ is non-trivial in $A_{D_{4}}$.

Let $r=\left(t, d_{1}, d_{2}, d_{3}\right) \in L_{-}$be a primitive vector with $t \in\langle 2\rangle^{\oplus 2}$ and $d_{i} \in D_{4}$, $i=1,2,3$, according to the direct sum decomposition of $L_{-}$given in Lemma 2.2. It follows from the previous remark that we have these cases:

i) if $d_{1}, d_{2}, d_{3}$ are divisible by two, then $t$ is not divisible by two, hence $r / 2=t / 2$ is not trivial in $A_{L_{-}}$;

ii) if at least one among $d_{1}, d_{2}, d_{3}$ is not divisible by two and not all its coefficients are odd, then $r+\sigma^{*}(r) / 2$ is non-trivial in $A_{L_{-}}$;

iii) if at least one among $d_{1}, d_{2}, d_{3}$ is not divisible by two and the non-two divisible $d_{i}$ 's all have odd coefficients, then $r / 2$ is in $L_{-}^{*}$ and it is non-trivial in $A_{L_{-}}$.

Hence $m_{r} \in\{1,2\}$.

We now characterize the remaining cases. According to the previous discussion, there are two possibilities:

i) if $r / 2 \in A_{L_{-}}$i.e. $\left(r, L_{-}\right)=2 \mathbb{Z}$, then

$$
M_{r}=\left\langle r / 2, \sigma^{*}(r) / 2\right\rangle \text {. }
$$

Since $\Lambda_{r}$ is primitive in $L_{-}$, we have that $\sigma^{*}(r) / 2 \neq r / 2$ in $A_{L_{-}}$. In particular $n$ is not even, since otherwise $q(r / 2)=-n / 2 \in \mathbb{Z}$ (hence $\sigma^{*}(r) / 2=r / 2$ in $A_{L_{-}}$by Lemma 2.6). Therefore $m_{r}=2$.

ii) if $r / 2 \notin A_{L_{-}}$i.e. $\left(r, L_{-}\right)=\mathbb{Z}$, then $m_{r}=1$ and

$$
M_{r}=\left\langle\left(r+\sigma^{*}(r)\right) / 2\right\rangle \text {. }
$$

We call $m_{r}$ the index of the Heegner divisor $\mathcal{D}_{r}, r \in L_{-}$.

Proposition 3.4 (Existence).

i) There exist Heegner divisors of type $n$ and index 1 for every $n \geq 1$;

ii) there exist Heegner divisors of type $n$ and index 2 for every odd $n \geq 1$.

(Note that the case of even type and index 2 is excluded by Proposition 3.3).

Proof. Consider the following vectors in $L_{-}$:

$$
\begin{aligned}
& r_{1}(1)=f_{1}^{1}+f_{1}^{2}, \quad r_{1}(k)=(k-1) t_{1}+k f_{1}^{1}+f_{1}^{2} \quad(k>1), \\
& r_{2}(k)=k t_{1}+(k+1) f_{1}^{1}, \\
& r_{3}(k)=(k-1) t_{1}+k f_{1}^{1}+f_{1}^{2}+f_{1}^{3}, \\
& r_{4}(k)=t_{1}+2 k t_{2}+(k+1)\left(f_{1}^{1}+f_{2}^{1}\right)+k\left(f_{1}^{2}+f_{2}^{2}\right),
\end{aligned}
$$

with respect to the basis of $L_{-}$defined in section 2 . It is easy to check that the rank two lattice $\Lambda_{i}(k)=\left\langle r_{i}(k), \sigma^{*}\left(r_{i}(k)\right)\right\rangle$ is primitive in $L_{-}$, since the rank two minors of the matrix with rows $r_{i}(k), \sigma^{*}\left(r_{i}(k)\right)$ have no common factor. Moreover, notice that

$$
r_{i}(k)^{2}=\left\{\begin{array}{lll}
-2(2 k) & \text { if } \quad i=1 \\
-2(2 k+1) & \text { if } \quad i=2,3 \\
-2(4 k+1) & \text { if } \quad i=4
\end{array}\right.
$$


in particular the Heegner divisor $\mathcal{D}_{r_{i}(k)}$ has even type $n=2 k$ for $i=1$ and odd type otherwise. By Proposition 3.3 the Heegner divisor $\mathcal{D}_{r_{i}(k)}$ has index 1 if $i=1,3$ since $\left(r_{i}(k), L_{-}\right)=\mathbb{Z}$, hence $\left.\mathrm{i}\right)$ is proved.

For $i=2$ it can be easily checked that the index is 2 iff $k$ is odd i.e. the type $n=2 k+1 \equiv 3(\bmod 4)$. For $i=4$ the type is $n=4 k+1 \equiv 1(\bmod 4)$ and the index is 2 by Proposition 3.3, hence ii) is proved.

The following remark will be useful in the next section

Lemma 3.5. Let $\mathcal{D}_{r}$ be a Heegner divisor of even type, then $\tilde{k} / 2 \notin M_{r}$.

Proof. Since $\mathcal{D}_{r}$ has index 1 by Proposition 3.3 we have $M_{r}=\left\langle\left(r+\sigma^{*}(r)\right) / 2\right\rangle$. From Lemma 2.5 it follows that the isomorphism $\gamma: A_{L_{+}} \rightarrow A_{L_{-}}$sends $\tilde{k} / 2$ to

$$
\gamma(\tilde{k} / 2)=(1 / 2,1 / 2,0, \ldots, 0)
$$

(in the usual coordinates for $L_{-}$). Consider a vector:

$$
r=\left(h_{1}, h_{2}, a_{1}, \ldots, a_{4}, b_{1}, \ldots, b_{4}, c_{1}, \ldots, c_{4}\right),
$$

such that $\Lambda_{r}$ is primitive in $L_{-}$and assume that $\left(r+\sigma^{*}(r)\right) / 2=\gamma(\tilde{k} / 2)$ in $A_{L_{-}}$. This means that:

i) $h_{1}$ and $h_{2}$ are odd,

ii) $a_{3}, b_{3}$ and $c_{3}$ are even,

iii) $a_{1}+a_{2}+a_{4}, b_{1}+b_{2}+b_{4}$ and $c_{1}+c_{2}+c_{4}$ are even.

In this case it can be easily checked that $\left(r, L_{-}\right)=2 \mathbb{Z}$, giving a contradiction by Proposition 3.3.

\section{HeEgner Divisors AND Genus 3 CURVES}

Let $X_{r}$ be the generic $K 3$ surface with period point in a Heegner divisor $\mathcal{D}_{r}$, then its Picard number is equal to 10 and

$$
L_{+} \oplus \Lambda_{r} \subset \operatorname{Pic}\left(X_{r}\right) .
$$

In this section we describe the loci of genus three curves corresponding to Heegner divisors of given type and index. We first recall the special role of Heegner divisors of type 1 in Kondō's construction.

\subsection{Type 1.}

Lemma 4.1. A period point in $\mathcal{M}$ belongs to a Heegner divisor of type 1 iff the corresponding K3 surface is not the four cyclic cover of the plane branched along a smooth plane quartic.

Proof. See Theorem 2.5, [7].

Proposition 4.2. The mirrors $\mathcal{D}_{n}, \mathcal{D}_{h}$ are the unique Heegner divisors of type 1:

- $\mathcal{D}_{n}$ has index 1 , and its generic point corresponds to a plane quartic with a node;

- $\mathcal{D}_{h}$ has index 2, and its generic point corresponds to a smooth hyperelliptic genus three curve.

Proof. By Lemma 3.3, Theorem 4.3 and Theorem 5.4 in [7]. 
Remark 4.3. i) In [1] it is proved that the period map $\mathcal{P}$ can be defined on the blowup of the GIT moduli space of semistable plane quartics in one point. This period map gives an isomorphism between $\mathcal{D}_{n}$ and the locus of stable singular quartics. Besides, it is proved that the Baily-Borel compactification of $\mathcal{D}_{h}$ is isomorphic to the GIT moduli space of semistable sets of eight unordered points in $\mathbb{P}^{1}$ (see also [6]).

ii) The transcendental lattices of the generic $K 3$ surface in $\mathcal{D}_{n}$ and $\mathcal{D}_{h}$ are given respectively by:

$$
\begin{aligned}
& T_{n} \cong U^{\oplus 2} \oplus A_{1}^{\oplus 8}, \\
& T_{h} \cong U(2)^{\oplus 2} \oplus D_{8} .
\end{aligned}
$$

\subsection{Type $n>1$.}

Definition 4.4. Let $C$ be a plane quartic and let $\pi: X_{C} \longrightarrow \mathbb{P}^{2}$ be the four cyclic cover of the plane branched along $C$. An irreducible plane curve $D$ is a splitting curve for $C$ if the inverse image $\pi^{-1}(D)$ is the union of four distinct curves.

It is easy to see that a smooth plane curve $D$ is a splitting curve for $C$ if and only if the restricted cover $\pi_{\mid \pi^{-1}(D)}$ is trivial. If the curve is not smooth it can happen that it is partially normalized by the cover $\pi$ i.e. the four curves in $\pi^{-1}(D)$ are not isomorphic to $D$ and they can intersect each other outside the ramification curve. We will see examples of this behaviour in the last section.

With $X_{r}$ as before, we call $\pi: X_{r} \longrightarrow \mathbb{P}^{2}$ the four cyclic cover of $\mathbb{P}^{2}, C_{r}$ the corresponding branch quartic and $\pi_{2}: X_{r} \longrightarrow S_{r}$ the associated double cover of the Del Pezzo surface $S_{r}$ (see section 2). We start with a simple remark

Lemma 4.5. If $\mathcal{D}_{r}$ is a Heegner divisor of type $n>1$, then $C_{r}$ admits a splitting curve of even degree.

Proof. As noticed before, we have $L_{+} \oplus \Lambda_{r} \subset \operatorname{Pic}\left(X_{r}\right)$. Consider a class of the form $w=\tilde{x}+r$ where $\tilde{x} \in L_{+}$with $\tilde{x}^{2} \geq 2 n-2$. Then $w^{2} \geq-2$, hence $w$ or $-w$ is effective by the Riemann Roch Theorem. Assume that $w$ is effective and write $w=\sum a_{i} w_{i}$, where $a_{i}$ are positive integers and $w_{i}$ are irreducible curves. Then we can assume that $w_{1}=\tilde{x}_{1}+r_{1}$ with $r_{1} \neq 0$. The $\sigma^{*}$-orbit of $w_{1}$ clearly contains four distinct elements: $w_{1}, \sigma^{*}\left(w_{1}\right), \tau^{*}\left(w_{1}\right)=\tilde{x}_{1}-r_{1},\left(\sigma^{*}\right)^{3}\left(w_{1}\right)=\sigma^{*}\left(\tilde{x}_{1}\right)-\sigma^{*}\left(w_{1}\right)$. Hence the image $\pi(R)$ of a curve $R$ in the class $w_{1}$ is a splitting curve for $C$. The degree of $R$ is given by $\operatorname{deg}(R)=\left(\tilde{x}_{1},-\tilde{k}\right)=2\left(x_{1},-k\right)$.

Theorem 4.6. Let $\mathcal{D}_{r}$ be a Heegner divisor of type $n>1$ (so $r \in L_{-}, r^{2}=-2 n$ ) with index $m_{r} \in\{1,2\}$. Then there exists a rational splitting curve $D$ for $C_{r}$ with the following properties:

1)

$$
\operatorname{deg}(D)= \begin{cases}2(n-1) & \text { if } m_{r}=1 \\ n-2 & \text { if } m_{r}=2 .\end{cases}
$$

2) The inverse image $\pi_{1}^{-1}(D)$ in $S_{r}$ is the union of two smooth rational curves (hence $\pi^{-1}(D)$ is the union of four smooth rational curves in $X_{r}$ ).

Proof. The idea is to construct the class of a (-2)-curve in $\operatorname{Pic}\left(X_{r}\right) \backslash L_{+} \oplus \Lambda_{r}$ starting from the information given by the structure of the group $M_{r}$. 
i) If $m_{r}=1$ (i.e. $M_{r} \cong \mathbb{Z}_{2}$ ), then there exists $\tilde{x} \in L_{+}$such that:

$$
(\tilde{x}+f) / 2 \in \operatorname{Pic}\left(X_{r}\right)
$$

where $f=r+\sigma^{*}(r)$. Notice that $\left(\tilde{x}^{\prime}+f\right) / 2 \in \operatorname{Pic}\left(X_{r}\right)$ for any $\tilde{x}^{\prime} \in L_{+}^{*}$ with $\tilde{x}^{\prime} / 2=\tilde{x} / 2$ in $A_{L_{+}}$. Moreover

$$
q(\tilde{x} / 2)=-q(f / 2)=n .
$$

Then $\tilde{x} / 2 \in A_{L_{+}}$belongs to the class $C_{3}$ in Lemma 2.6 if $n$ is odd and to the class $C_{4}$ if $n$ is even.

If $n$ is odd we can assume (up to the action of the Weyl group; see Lemma 2.7 and Lemma 3.5) that:

$$
\tilde{x} / 2=\left(\tilde{e}_{1}-\tilde{e}_{2}\right) / 2 \in A_{L_{+}} .
$$

Let $x^{\prime}=(n-1) e_{0}-(n-2) e_{1}-e_{2} \in \operatorname{Pic}\left(S_{r}\right)$, then $\tilde{x}^{\prime} / 2=\tilde{x} / 2$ in $A_{L_{+}}$. Hence by a previous remark

$$
y=\left(\tilde{x}^{\prime}+f\right) / 2 \in \operatorname{Pic}\left(X_{r}\right)
$$

and $y^{2}=\left(x^{\prime}\right)^{2} / 2-n=-2$. Notice that $y+\tau^{*}(y)=x^{\prime}$ and the arithmetic genus $p_{a}\left(x^{\prime}\right)$ is zero by the adjunction formula.

The class $y$ can be written as $y=\sum_{i=1}^{l} a_{i} y_{i}$, where $a_{i}$ are positive integers and $y_{i}$ are irreducible curves. Since $p_{a}\left(x^{\prime}\right)=0$, there exists an irreducible component $y_{1}$ of $y$ which is a smooth rational curve and doesn't belong to $L_{+}$. This can be written as

$$
y_{1}=\left(\tilde{x}_{1}+f_{1}\right) / 2, \quad \tilde{x}_{1} \in L_{+}, f_{1} \in \Lambda_{r}, f_{1} \neq 0 .
$$

Since $y^{2}=y_{1}^{2}=-2$ and $f_{1}^{2} \leq f^{2}$ we have $x_{1}^{2} \geq\left(x^{\prime}\right)^{2}=2(n-2)$. Moreover, since $x_{1}$ is a component of $x^{\prime}$, its arithmetic genus is zero. This gives

$$
0=p_{a}\left(x_{1}\right)=\frac{x_{1}^{2}+\left(x_{1}, k\right)}{2}+1 \geq \frac{2(n-2)+\left(x_{1}, k\right)}{2}+1,
$$

hence

$$
\left(y_{1},-k\right)=\left(x_{1},-k\right) \geq 2(n-1)=(y,-k) .
$$

This implies that $y_{1}=y$ i.e. $y$ is a $(-2)$-curve. If $R$ be the curve in the class $y$, then the plane curve $D=\pi(R)$ is a rational splitting curve for $C_{r}$ and:

$$
\operatorname{deg}(D)=-\left(x^{\prime}, k\right)=2(n-1) .
$$

If $n$ is even, then we can assume (up to the action of the Weyl group; see Lemma 2.7) that:

$$
\tilde{x} / 2=\left(\tilde{k}+2 \tilde{e}_{0}-\sum_{i} \tilde{e}_{i}+\tilde{e}_{1}\right) / 2=\left(\tilde{e}_{0}-\tilde{e}_{1}\right) / 2 \in A_{L_{+}} .
$$

The proof follows as in the odd case with the choice $x^{\prime}=(n-1) e_{0}-e_{1}-(n-2) e_{2}$. ii) If $m_{r}=2$ (i.e. $M_{r} \cong \mathbb{Z}_{2} \oplus \mathbb{Z}_{2}$ ), then there exists $\tilde{x} \in L_{+}$such that:

$$
(\tilde{x}+r) / 2, \sigma^{*}(\tilde{x}+r) / 2 \in \operatorname{Pic}\left(X_{r}\right),
$$

where

$$
q(\tilde{x} / 2)=-q(r / 2)=n / 2 .
$$

Then $\tilde{x} / 2 \in A_{L_{+}}$belongs to the class $C_{1}$ in Lemma 2.6 if $n \equiv 3(\bmod 4)$ and to the class $C_{2}$ if $n \equiv 1(\bmod 4)$.

If $n \equiv 3(\bmod 4)$, then we can assume that:

$$
\tilde{x} / 2=\tilde{e}_{1} / 2 .
$$


Let $n=4 s+3$ and consider the vector $x^{\prime}=2 s e_{0}-(2 s-1) e_{1}$. Note that $p_{a}\left(x^{\prime}\right)=0$ by the adjunction formula. Then

$$
y=\left(x^{\prime}+r\right) / 2 \in \operatorname{Pic}\left(X_{r}\right)
$$

and $y^{2}=-2$.

It can be proved as in i) that $y$ is a smooth $(-2)$-curve and if $R$ is a curve representing $y$, then $D=\pi(R)$ is a rational splitting curve with

$$
\operatorname{deg}(D)=-\left(x^{\prime},-k\right)=n-2 .
$$

If $n \equiv 1(\bmod 4)$, then we can assume that:

$$
\tilde{x} / 2=\tilde{e}_{0} / 2 .
$$

Let $n=4 s+1$ and consider the class $x^{\prime}=(s-1)\left(e_{0}-e_{1}\right)$. Defining $y$ as above, similar computations give a rational splitting curve $D$ with:

$$
\operatorname{deg}(D)=-\left(x^{\prime}, k\right)=n-2 .
$$

Remark 4.7. Note that in the proof of Theorem 4.6 (the classes of) the preimages of the splitting curves in the Del Pezzo surface are given explicitly up to the action of the Weyl group.

Corollary 4.8. The Picard lattice of the $K 3$ surface $X_{r}$ is generated by $L_{+}$and two smooth rational curves $R, \sigma^{*}(R)$ with $R \notin L_{+} \oplus \Lambda_{r}$.

Proof. Obviously $\operatorname{Pic}\left(X_{r}\right) \supset\left\langle L_{+}, R, \sigma^{*}(R)\right\rangle$. Let $R=(\tilde{x}+y) / 2$, where $\tilde{x} \in L_{+}$ and $y \in \Lambda_{r}$ are primitive. Then the thesis follows since the embedding of $L_{+}$in $\operatorname{Pic}\left(X_{r}\right)$ is primitive and the classes $R, \sigma^{*}(R)$ are not divisible in $L_{K 3}$.

We also give a partial converse to the previous result:

Proposition 4.9. Let $C$ be a plane quartic admitting a rational splitting curve $D$ such that the inverse image of $D$ in $S_{C}$ is the union of two smooth rational curves with primitive classes in Pic $\left(S_{C}\right)$. Let $d$ be the minimal degree of a curve $D$ with this property. Then $X_{C}$ belongs to a Heegner divisor

i) of index $m=2$ and type $n=d+2$ if $d$ is odd;

ii) of index $m=1$ and type $n=(d+2) / 2$ if $d$ is even.

Proof. Let $R$ be one of the inverse images of $D$ in $X_{C}$. Notice that:

$$
R=(x+y) / 2 \in \operatorname{Pic}\left(X_{C}\right),
$$

where

$$
x=R+\tau^{*}(R) \in L_{+}, y=R-\tau^{*}(R) \in L_{-} \cap \operatorname{Pic}\left(X_{C}\right) .
$$

An easy computation gives:

$$
x^{2}=2(d-2), y^{2}=-2(d+2) .
$$

Notice that $y \neq 0$ in $L_{-}$since otherwise $x=2 R$ (which is the class of a preimage of $D$ in $S_{C}$ ) would be divisible in $L_{+}$. Thus $X_{C}$ belongs to a Heegner divisor $\mathcal{D}_{r}$ with $y \in \Lambda_{r}$. Let $\Lambda_{r}^{\perp}$ be the orthogonal complement of $\Lambda_{r}$ in $L_{-}$and $P_{r}$ be the orthogonal complement of $\Lambda_{r}^{\perp}$ in $L_{K 3}$. Then we define

$$
M_{r}=P_{r} / L_{+} \oplus \Lambda_{r}
$$

Notice that:

$$
\sum_{i=0}^{3}\left(\sigma^{*}\right)^{i}(R)=-d \tilde{k}
$$


Then we have the following cases:

i) If $d$ is odd, then:

$$
\sum_{i=0}^{3}\left(\sigma^{*}\right)^{i}(R) / 2=\left(x+\sigma^{*}(x)\right) / 2=\tilde{k} / 2 \neq 0 \in A_{L_{+}} .
$$

Hence $x / 2 \neq \sigma^{*}(x) / 2 \in A_{L_{+}}$. Therefore

$$
M_{r} \cong\left\langle x / 2, \sigma^{*}(x) / 2\right\rangle \subset A_{L_{+}} ;
$$

this means that $\mathcal{D}_{r}$ has index $m_{r}=2$. By Theorem 4.6 (see also the proof) the curve $C$ admits a rational splitting curve $D^{\prime}$ of degree $d^{\prime}=-r^{2} / 2-2$ with smooth and primitive preimages in the Del Pezzo surface such that $\pi^{*}\left(D^{\prime}\right)=$ $\sum_{i=1}^{4}\left(\sigma^{*}\right)^{i}\left(R^{\prime}\right), i=1, \ldots, 4$, with $R^{\prime}-\tau^{*}\left(R^{\prime}\right)=r$. Then:

$$
\left(R^{\prime}-\tau^{*}\left(R^{\prime}\right)\right)^{2}=-2\left(d^{\prime}+2\right)
$$

is maximal i.e. $d^{\prime}$ is minimal. Then $d=d^{\prime}$ and $\mathcal{D}_{r}$ has type $n=d+2$.

ii) If $d$ is even, then:

$$
\sum_{i}\left(\sigma^{*}\right)^{i}(R) / 2=\left(x+\sigma^{*}(x)\right) / 2=0 \in A_{L_{+}} .
$$

Hence:

$$
x / 2=\sigma^{*}(x) / 2 \in A_{L_{+}} .
$$

The case $x / 2=0$ in $A_{L_{+}}$(i.e. $x=2 x^{\prime} \in L_{+}$) can be excluded since otherwise the inverse images of $D$ in $S_{C}$ would belong to divisible classes. Then the Heegner divisor $\mathcal{D}_{r}$ has index $m_{r}=1$ :

$$
M_{r}=\langle x / 2\rangle \subset A_{L_{+}} .
$$

By applying Theorem 4.6 as in i), the minimality condition on the degree gives that $\mathcal{D}_{r}$ has type $n=(d+2) / 2$.

Corollary 4.10. A plane quartic $C$ admits a splitting curve of odd degree if and only if $X_{C}$ belongs to a Heegner divisor of index 2.

Proof. The result follows from Theorem 4.6 and the proof of Proposition 4.9 if one notices that in the odd degree case the inverse images of the splitting curve in $\operatorname{Pic}\left(S_{C}\right)$ always have primitive classes.

Remark 4.11. i) Splitting curves of even degree do not always give rise to Heegner divisors. From the proof of Proposition 4.9 it follows that this happens iff a inverse image $R$ of the splitting curve in the $K 3$ surface satisfies:

$$
R-\tau^{*}(R)=0 .
$$

For example, let $C$ be the generic plane quartic with equation $f_{4}(x, y, z)=0$. It is easy to prove that a plane quartic $D$ of the form $l(x, y, z)^{4}=f_{4}(x, y, z)$, where $l$ is linear, is a splitting curve for $C$. The inverse image of $D$ in $X_{C}$ splits in four genus three curves $\left(\sigma^{*}\right)^{i}(R), i=0, \ldots, 3$, which are hyperplane sections of $X_{C}$ in $\mathbb{P}^{3}$ (i.e. they all belong to the class $\left.-\tilde{k} \in L_{+}\right)$.

ii) If the splitting curve has even degree $d \not \equiv 2(\bmod 4)$ an easy computation shows that its inverse images have primitive classes in the Del Pezzo surface.

iii) Theorem 4.6 and Proposition 4.9 describe the loci of plane quartics corresponding to the union of all Heegner divisors of given type and index. However, it is not clear if these loci are irreducible. In other words, it is unknown if a Heegner divisor 
is determined uniquely by its type and index. Note that this problem is equivalent to classifying the embeddings of the lattice $\Lambda_{n}$ in $L_{-}$up to the action of $\Gamma$.

iv) In [6] Heegner divisors in $\mathcal{D}_{h}$ (in fact, this is a union of our Heegner divisors with fixed type and index intersected with $\mathcal{D}_{h}$ ) are introduced and the existence of an automorphic form vanishing exactly on them is proved.

\section{EXAMPLES}

In this section we give some examples of Heegner divisors corresponding to plane quartics with splitting curves of degree one, two and three. We will usually identify Heegner divisors in $\mathcal{M}$ and their inverse image by $\mathcal{P}$ in $\mathcal{M}_{3}$.

Let $C \subset \mathbb{P}^{2}$ be a plane curve of degree $d d^{\prime}$ with $d=2,4$ and let $\pi_{d}: Z \rightarrow \mathbb{P}^{2}$ be the $d: 1$ cover of $\mathbb{P}^{2}$ branched along $C$. We start recalling the following result:

Proposition 5.1 (Proposition 1.7, Ch.3, [12]). Let $D \subset \mathbb{P}^{2}$ be a curve not containing components of $C$. Let $\tilde{D}=\pi_{d}^{-1}(D)$. The restriction of the cover:

$$
\pi_{d \mid \tilde{D}}: \tilde{D} \longrightarrow D
$$

is trivial if and only if there exists a curve $B \subset \mathbb{P}^{2}$ of degree $d^{\prime}$ such that:

$$
C \cdot D=d B \cdot D \text {. }
$$

By the remark after Definition 4.4, this result characterizes smooth splitting curves of $C$.

5.1. Quartics with a hyperflex. Let $C$ be a generic smooth plane quartic with a hyperflex line $L$ i.e.

$$
L \cdot C=4 p, \quad p \in C .
$$

After a projective transformation we can assume that the line $L$ is given by the equation $x=0$ and that the quartic $C$ is of the form:

$$
C: x f_{3}(x, y, z)+z^{4}=0
$$

where $f_{3}$ is a cubic polynomial in $\mathbb{C}[x, y, z]$. It is known that the locus of plane quartics with at least one hyperflex line is an irreducible closed subvariety of codimension one in the moduli space of genus three curves (see Proposition 4.9, Ch.I, [12]). As usual, let $\pi_{1}: S_{C} \rightarrow \mathbb{P}^{2}$ and $\pi: X_{C} \rightarrow \mathbb{P}^{2}$ be the double and the $4: 1$ cover of $\mathbb{P}^{2}$ branched along $C$ respectively.

Proposition 5.2. The locus of plane quartics with a hyperflex line is the unique Heegner divisor of type 3 and index 2.

Proof. Let $C$ be a plane quartic with a hyperflex line $L$. By Proposition 5.1 both $\pi_{1}$ and $\pi$ are trivial over $L$. Hence the line $L$ is a splitting curve for $C$, moreover it splits in the union of two smooth rational curves on the Del Pezzo surface $S_{C}$. By Proposition 4.9 it follows that $X_{C}$ belongs to a Heegner divisor of type $n=3$ and index 2 (the degree of $L$ is obviously minimal). The converse follows from Theorem 4.6. Since the locus of plane quartics with a hyperflex line is irreducible in $\mathcal{M}_{3}$, there is only one Heegner divisor of type 3 and index 2 .

We denote this Heegner divisor with $\mathcal{D}_{\text {flex }}$ and with $X$ the generic $K 3$ surface in it. 
Let $M, M^{\prime}$ be the inverse images of $L$ in the Del Pezzo surface $S$. Each of them splits in the union of two smooth rational curves on $X$ :

$$
\pi_{2}{ }^{*}(M)=M_{1}+M_{2}, \pi_{2}{ }^{*}\left(M^{\prime}\right)=M_{1}^{\prime}+M_{2}^{\prime}
$$

Notice that

$$
M_{1}+M_{2} \in L_{+}, M_{1}-M_{2} \in L_{-} \cap \operatorname{Pic}(X) .
$$

If $r=M_{1}-M_{2}$, it is easy to check that $r^{2}=-6$. Hence the period point of $X$ lies in the hyperplane section of $B$ determined by the vector $r \in L_{-}$. By Corollary 4.8 we have:

$$
\operatorname{Pic}(X)=\left\langle L_{+}, M_{1}, M_{1}^{\prime}\right\rangle .
$$

Notice that $M, M^{\prime}$ are two (-1)-curves on $S$ with:

$$
M+M^{\prime}=\pi_{1}^{*}(L)=-k .
$$

Hence we can assume, up to the action of the Weyl group, that:

$$
M=e_{7}, \quad M^{\prime}=3 e_{0}-\left(e_{1}+\cdots+e_{6}+2 e_{7}\right) .
$$

Then the intersection matrix of the Picard lattice with respect to the basis $\tilde{e}_{0}, \ldots, \tilde{e}_{7}$, $M_{1}, M_{1}^{\prime}$ can be easily computed.

5.2. Quartics with splitting conics. We consider the locus of smooth plane quartics $C$ such that there exists an irreducible conic $T$ with intersection divisor of the form:

$$
T \cdot C=4 p+4 q,
$$

where $p \neq q$. After a projective transformation we can assume that the conic $T$ is given by:

$$
T: x y-z^{2}=0
$$

and that $p=(0,1,0), q=(1,0,0)$. Then, up to projectivities, the equation of the quartic $C$ is of the form:

$$
C:\left(x y-z^{2}\right) f_{2}(x, y, z)+z^{4}=0,
$$

where $f_{2}$ is a quadratic polynomial in $\mathbb{C}[x, y, z]$. It is known (see [5]) that a smooth plane quartic $C$ has 63 one dimensional families of tangent conics and that each family contains six reducible conics (i.e. the union of two bitangents). We now prove that

Lemma 5.3. The locus of plane quartics with an irreducible splitting conic is a codimension one closed irreducible subvariety of $\mathcal{M}_{3}$.

Proof. Let $\Delta \subset \mathbb{P}^{14}$ be the discriminant locus corresponding to singular plane quartics. Notice that polynomials like the one defining $C$ give a 6 dimensional irreducible subvariety $A$ of $\mathbb{P}^{14} \backslash \Delta$. Let $\phi: \mathbb{P}^{14} \backslash \Delta \rightarrow \mathcal{M}_{3} \backslash \mathcal{M}_{3}^{h}$ be the natural morphism to the moduli space of non-hyperelliptic genus three curves. This is a closed and surjective morphism (see Proposition 4.7, [12]). In particular, $V=\phi(A)$ is an irreducible closed subvariety of $\mathcal{M}_{3} \backslash \mathcal{M}_{3}^{h}$. Note that the dimension of the fibers of $\phi$ over $V$ is equal to one. In fact, it equals the dimension of:

$$
\{g \in P G L(3, \mathbb{C}): g(p)=p, g(q)=q, g(T)=T\} .
$$

Thus $V$ has codimension one in $\mathcal{M}_{3} \backslash \mathcal{M}_{3}^{h}$. In fact, it can be proved as in [12] that $V$ is also closed in $\mathcal{M}_{3}$. 
Similarly to Proposition 5.2 it can be proved

Proposition 5.4. The locus of plane quartics with an irreducible splitting conic is the unique Heegner divisor of type 2 and index 1.

We denote this divisor with $\mathcal{D}_{\text {con }}$. As in the previous example, the Picard lattice of the generic $K 3$ surface in $\mathcal{D}_{\text {con }}$ can be easily computed.

5.3. Quartics with splitting cubics. A simple remark is the following:

Lemma 5.5. A plane quartic $C$ has a hyperflex line if and only if there exists a cubic $D$ such that the 4:1 cover of $\mathbb{P}^{2}$ branched along $C$ is trivial over $D$.

Proof. Notice that a quartic $C$ has a hyperflex line $L$ if and only if there is a point $p \in C$ with

$$
4 p \in\left|O_{C}(1)\right|=\left|K_{C}\right| .
$$

In this case, let $M$ be a line through $p, M \neq L$, then $M \cdot C-p=p_{1}+p_{2}+p_{3}$. Hence

$$
4\left(p_{1}+p_{2}+p_{3}\right) \in\left|4 K_{C}-4 p\right|=\left|3 K_{C}\right|
$$

i.e. there exists a cubic curve $D$ intersecting $C$ with multiplicity 4 in each point and such that the points in $C \cdot D$ lie on the line $M$. Equivalently, by Proposition 5.1 , the $4: 1$ cover of $\mathbb{P}^{2}$ branched along $C$ is trivial over $D$.

Conversely, if there exists a cubic $D$ with $D \cdot C=4\left(p_{1}+p_{2}+p_{3}\right)$ and a line $M$ with $M \cdot D=p_{1}+p_{2}+p_{3}$, then

$$
M \cdot C=p_{1}+p_{2}+p_{3}+p,
$$

hence $4 p=4 M \cdot C-D \cdot C \in\left|O_{C}(1)\right|$ i.e. $p$ is a hyperflex point.

Remark 5.6. In fact, Lemma 5.5 follows immediately if we recall that, up to a projective transformation, the equation of a quartic $C$ with a hyperflex line is of the form:

$$
x f_{3}(x, y, z)+z^{4}=0
$$

where $f_{3}$ is a cubic polynomial in $\mathbb{C}[x, y, z]$.

In general, a (singular) plane cubic $D$ can be a splitting curve for a quartic $C$ even if the 4:1 cover branched along $C$ is not trivial over $D$ i.e. the irreducible components of $\pi^{-1}(D)$ are not isomorphic to $D$. Let $\pi: X_{C} \rightarrow \mathbb{P}^{2}$ and $\pi_{1}: S_{C} \rightarrow \mathbb{P}^{2}$ be the $4: 1$ and the double cover branched along $C$ respectively.

Proposition 5.7. Let $C$ be a plane quartic with a splitting nodal cubic D. Then $X_{C}$ belongs to a Heegner divisor of index 2 and

i) type 3 if $\pi$ is trivial over $D$ (i.e. $X_{C}$ lies in $\mathcal{D}_{\text {flex }}$ ),

ii) type 7 if $\pi_{1}$ is trivial over $D$,

iii) type 5 otherwise.

Proof. Up to projectivities we can assume that $D$ is defined by the equation:

$$
y^{2}=x^{3}+x^{2} .
$$

A parametrization for $D$ is given by:

$$
\phi: \mathbb{C} \longrightarrow D, t \longmapsto\left(t^{2}-1, t\left(t^{2}-1\right)\right),
$$

where $\phi(1)=\phi(-1)$ is the node of $D$. 
The plane quartic $C$ with equation $f_{4}(x, y, z)=0$ intersects $D$ in the points $\phi(t)$, with:

$$
f_{4}\left(t^{2}-1, t\left(t^{2}-1\right), 1\right)=0 .
$$

In particular, if the intersection multiplicity of each point in $C \cap D$ is a multiple of four, then:

$$
f_{4}\left(t^{2}-1, t\left(t^{2}-1\right), 1\right)=q^{4},
$$

where $q \in \mathbb{C}[t]$ and $q^{4} \in \mathbb{C}\left[t^{2}-1, t\left(t^{2}-1\right)\right]$. It can be easily proved that

$$
\mathbb{C}\left[t^{2}-1, t\left(t^{2}-1\right)\right]=\{p \in \mathbb{C}[t]: p(1)=p(-1)\} \text {. }
$$

Hence:

$$
q(1)=i^{a} q(-1), \quad a=0, \ldots, 3 .
$$

The 4:1 cover of the plane branched along $C$ is given by:

$$
X_{C}=\left\{w^{4}=F(x, y, z)\right\} \subset \mathbb{P}^{3} .
$$

Notice that the map $\phi$ lifts to four distinct maps to $X_{C}$ :

$$
\phi_{k}: \mathbb{C} \longrightarrow X_{C}, \quad t \mapsto\left(t^{2}-1: t\left(t^{2}-1\right): 1: i^{k} q(t)\right),
$$

hence the inverse image of $D$ in $X_{C}$ has four isomorphic irreducible components:

$$
D_{k}=\phi_{k}(\mathbb{C}), \quad a=0, \ldots, 3 .
$$

We now study the restriction of the cover $\pi$ to $D$ for all values of $a$.

i) If $a=0$, then $q(1)=q(-1)$, hence each component $D_{k}$ is a nodal curve and $\pi$ is trivial over $D$. By Lemma 5.5 the plane quartic $C$ has a hyperflex line and $X_{C}$ belongs to the Heegner divisor $\mathcal{D}_{\text {flex }}$.

ii) If $a=2$, then $q(1)=-q(-1)$, hence the curves $D_{k}$ are smooth rational curves. Note that $\phi_{0}(1)=\phi_{2}(-1)$ and $\phi_{0}(-1)=\phi_{2}(1)$, so $D_{0}$ and $D_{2}$ meet in two points over the singular point $p=(0: 0: 1) \in D$. Similarly, $D_{1}$ and $D_{3}$ meet in two points over $p$. In this case the double cover $\pi_{1}$ of $\mathbb{P}^{2}$ branched along $C$ is trivial over $D$, in particular the inverse image of $D$ in $S_{C}$ is the union of two singular curves. Note that:

$$
r=D_{0}-\tau^{*}\left(D_{0}\right)=D_{0}-D_{2} \in \operatorname{Pic}\left(X_{C}\right) \cap L_{-}
$$

and $r^{2}=-4-2 \cdot 2-2 \cdot 3=-14$. We have a lattice embedding:

$$
\Lambda=\left\langle r, \sigma^{*}(r)\right\rangle \subset \operatorname{Pic}\left(X_{C}\right) \cap L_{-} \cong A_{1}(n)^{\oplus 2} .
$$

In particular $-14=-2 n\left(a^{2}+b^{2}\right), a, b \in \mathbb{Z}$. Thus we have $n=7$ and $X_{C}$ belongs to a Heegner divisor of type 7 and index 2 .

iii) If $a=1$, then $q(1)=i q(-1)$, hence the curves $D_{k}$ are smooth. In this case we also have $\phi_{0}(1)=\phi_{1}(-1)$ and $\phi_{0}(-1)=\phi_{3}(1)$, so $D_{0}$ meets $D_{1}$ and $D_{3}$. Similarly, $D_{2}$ meets $D_{3}$ and $D_{1}$. In particular, the inverse image of $D$ in $S_{C}$ is the union of two smooth rational curves. Note that:

$$
r=D_{0}-\tau^{*}\left(D_{0}\right)=D_{0}-D_{2} \in \operatorname{Pic}\left(X_{C}\right) \cap L_{-}
$$

and

$$
r^{2}=-4-2 \cdot 3=-10
$$

Then:

$$
\Lambda=\left\langle r, \sigma^{*}(r)\right\rangle \subset \operatorname{Pic}\left(X_{C}\right) \cap L_{-} .
$$

In fact (since $-r^{2} / 2$ is prime and $n \neq 1$ ) we easily get that this is an equality. Hence $X_{C}$ belongs to a Heegner divisor of type 5 and index 2. This could also be 
proved by applying Proposition 4.9 (note that the degree is minimal since $m=2$ and we can assume that $C$ has no hyperflex lines).

iv) The case $a=3$ is analogous to case iii).

Remark 5.8. A similar description could be given more generally for any rational splitting curve.

\section{ACKNOWLEDGEMENTS}

I would like to thank B. van Geemen for introducing me to this subject and for several valuable suggestions. I also wish to thank A. Laface for many interesting discussions. Part of this work was done during a visit to the Queen's University (Kingston, Ontario). I thank the Mathematics Department for hosting me.

\section{REFERENCES}

[1] M. Artebani. A compactification of $\mathcal{M}_{3}$ via $K 3$ surfaces. preprint, 2005.

[2] W. Barth, C. Peters, and A. Van de Ven. Compact complex surfaces, volume 4 of Ergebnisse der Mathematik und ihrer Grenzgebiete (3). Springer-Verlag, Berlin, 1984. MR749574 (86c:32026)

[3] N. Bourbaki. Éléments de mathématique. Fasc. XXXIV. Groupes et algèbres de Lie. Chapitre IV: Groupes de Coxeter et systèmes de Tits. Chapitre V: Groupes engendrés par des réflexions. Chapitre VI: systèmes de racines. Hermann, Paris, 1968. MR0240238 (39:1590)

[4] I. Dolgachev and D. Ortland. Point sets in projective spaces and theta functions. Astérisque, (165):210 pp., 1988. MR1007155 (90i:14009)

[5] I. Hadan. Tangent conics at quartic surfaces and conics in quartic double solids. Math. Nachr., 210:127-162, 2000. MR1738983 (2001h:14044)

[6] S. Kondō. The moduli space of 8 points on $\mathbb{P}^{1}$ and automorphic forms, to appear in the Proceedings of the Conference "Algebraic geometry in honor of Igor Dolgachev". math.AG/0504233.

[7] S. Kondō. A complex hyperbolic structure for the moduli space of curves of genus three. $J$. Reine Angew. Math., 525:219-232, 2000. MR1780433 (2001j:14039)

[8] E. Looijenga. Compactifications defined by arrangements. I. The ball quotient case. Duke Math. J., 118(1):151-187, 2003. MR1978885 (2004i:14042a)

[9] D. Mumford, J. Fogarty, and F. Kirwan. Geometric Invariant Theory. Ergeb. Math. Grenzgeb. (2), 34, Springer, 1994. MR1304906 (95m:14012)

[10] V. V. Nikulin. Factor groups of groups of the automorphisms of hyperbolic forms with respect to subgroups generated by 2-reflections. J. Soviet Math., 22:1401-1475, 1983.

[11] V.V. Nikulin. Integral symmetric bilinear forms and its applications. Math. USSR Izv., 14:103-167, 1980.

[12] A.M. Vermeulen. Weierstrass points of weight two on curves of genus three. Ph.D. thesis, Universiteit van Amsterdam, 1983. MR715084 (84j:14036)

Dipartimento di Matematica, Università di Milano, via C. Saldini 50, 20133 Milano, ITALIA

E-mail address: michela.artebani@unimi.it

E-mail address: artebani@mat.unimi.it 